Recherches en didactique des langues et des cultures

Les cahiers de l'Acedle

$15-2 \mid 2018$

Quelles médiations en didactique des langues et des cultures?

\title{
Enjeux de la double médiation du plurilinguisme dans le milieu académique multilingue. Internationalisation ou diversité dans l'enseignement supérieur?
}

Patchareerat Yanaprasart

\section{CpenEdition}

Édition électronique

URL : http://journals.openedition.org/rdlc/2991

DOI : 10.4000/rdlc.2991

ISSN : $1958-5772$

Éditeur

ACEDLE

Référence électronique

Patchareerat Yanaprasart, «Enjeux de la double médiation du plurilinguisme dans le milieu académique multilingue. Internationalisation ou diversité dans l'enseignement supérieur? », Recherches en didactique des langues et des cultures [En ligne], 15-2 | 2018, mis en ligne le 02 juin 2018, consulté le 30 avril 2019. URL : http://journals.openedition.org/rdlc/2991 ; DOI : 10.4000/rdlc.2991

Ce document a été généré automatiquement le 30 avril 2019.

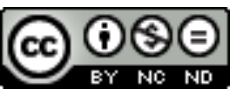

Recherches en didactique des langues et des cultures is licensed under a Creative Commons AttributionNonCommercial-NoDerivatives 4.0 International License 


\title{
Enjeux de la double médiation du plurilinguisme dans le milieu académique multilingue. Internationalisation ou diversité dans l'enseignement supérieur?
}

\author{
Patchareerat Yanaprasart
}

\section{Introduction}

1 Les transformations du contexte économique et sociopolitique ces dernières années ont amené des institutions d'enseignement supérieur à réfléchir sur les enjeux et les conséquences de l'internationalisation de la formation et de la recherche. Le monde académique et scientifique est en train de vivre une tension entre deux tendances opposées. D'une part, la diversité est un fait et son maintien est stipulé comme un principe de base dans le processus de Bologne, d'où l'encouragement à devenir plurilingue. D'autre part, la promotion de l'anglais comme lingua academica unique qui est pour certains de permettre une extraordinaire avancée de la connaissance alors que pour d'autres elle est conçue « comme condition d'une connaissance qui se veut universelle » (Berthoud, 2016: 167).

2 Pour Chaplier (2014), le développement et la promotion de l'anglais comme langue internationale pour les sciences et pour l'enseignement/apprentissage vont au-delà du problème de langue. Ces actions font apparaître des arguments aussi bien idéologiques que pragmatiques, sur son mode d'enseignement dans les systèmes éducatifs, sur des relations de pouvoir, des rapports conflictuels et des tensions sociales. Graddol (2006) a observé que les enseignants d'anglais de par le monde sont majoritairement « non natifs » et a estimé 2 milliards d'apprenants d'anglais en 2010. Ceci est d'autant plus vrai pour un nombre considérable d'enseignants auxquels il est demandé de communiquer, construire 
et transmettre des savoirs dans une langue qui n'est pas la leur, soit dans la langue internationale, dans la langue locale de l'institution ou dans la langue de la discipline. Ainsi, il y a des enseignants dont la langue première n'est pas la langue locale et qui enseignent dans la langue locale, des enseignants dont la langue première est la langue locale et qui enseignent dans une autre langue ou des enseignants dont la langue première n'est pas la langue locale et qui enseignent dans une autre langue. Leur point commun est qu'ils sont tous des enseignants non natifs de la langue d'enseignement.

Alors que leur nombre est en train de dépasser largement le nombre d'enseignants locaux natifs monolingues, le modèle du «native-speakerism » (Holliday, 2006) fonctionne-t-il toujours comme une sorte d'idéologie de l'authenticité du "locuteur natif idéal monolingue » ? Cette idéologie, n'est-elle pas en opposition avec le caractère multilingue et multiculturel de la société, voire l'environnement plurilingue où se trouvent des étudiants venant du monde entier et dans lequel ils cherchent à s'immerger ? D'autant plus que, selon Bologna Process, la diversité représente une condition sine qua non à la qualité de la formation, de la recherche et de la promotion institutionnelle. Aussi s'avère$\mathrm{t}$-il indispensable que chaque acteur puisse communiquer "efficacement» dans des situations multilingues et multiculturelles (Forster Vosicki, 2015). Une question se pose : qu'entend-on par efficacité dans le contexte d'internationalisation des marchés éducatifs? Parle-t-on d'une efficacité pédagogique et scientifique, d'une efficacité communicationnelle et administrative ou d'une efficacité commerciale - promotion, image et compétitivité ?

4 En effet, les enseignants, en tant que transmetteurs, vecteurs et négociateurs de savoirs, de langues et de cultures, sont dépendants des valeurs marchandes et symboliques des langues d'enseignement (Derivry, 2011). Pour Gajo (2005), les enseignants assument également les fonctions de déclencheur d'évaluation, de stimulateur de parole et de médiateur. Ils jouent les rôles de facilitateurs et d'accompagnateurs dans la découverte de nouvelles perspectives (Roussi et Cherkaoui Messin, 2011). Dans cette perspective, la question concerne moins ici l'impérialisme linguistique de l'anglais dans le monde (Phillipson, 1992) que qui utilise quelle langue, avec qui, pour quelle raison, dans quel contexte, à quel moment et de quelle manière. Comment l'usage d'une langue d'enseignement est encadré, soutenu, géré, valorisé, outillé, médié ou remédié et perçu? (Llurda, E., 2005 ; Dervin et Badrinathan, 2011)

5 En poursuivant leur objectif concurrentiel, les institutions universitaires font appel à la main d'œuvre hautement qualifiée, d'où une grande mobilité de professeurs expatriés non natifs de la langue locale dans le monde entier. De quelle manière cette identité 'nonnatif mais plurilingue' est-elle reconnue par ces professeurs (identité revendiquée) ou imposée par leurs collègues, leurs étudiants et leur institution ((identité assignée), Varghese, Morgan, Johnson et Johnson, 2005) ? Comment l'université perçoit-elle le plurilinguisme de sa communauté scientifique et institutionnelle (enrichissement, potentialité, restriction)? Quelles sont les mesures de soutien et d'encouragement pour mettre ou non le profil plurilingue en valeur? Quels arguments avancés par la direction dans la politique d'embauche? L'université souhaite-t-elle ou peut-elle répondre aux besoins académiques, professionnels et sociaux de l'ensemble de ses acteurs dans leur diversité ? La dimension linguistique, voire multilingue, fait-elle partie de la gestion de la diversité au sein de l'institution? Plus particulièrement, quelle politique linguistique universitaire à mettre en place pour trouver un juste équilibre entre l'anglais lingua academica et les autres langues, pour garantir une convergence entre 
l'internationalisation de la formation et le maintien de la diversité au niveau individuel et institutionnel, ce en termes de complémentarité et d'intégration? Bon nombre de questions méritent d'être approfondies.

6 En fin de compte, que signifie l'internationalisation en termes de défis pour les étudiants, les enseignants, les collaborateurs scientifiques et les administrateurs en général, et pour la communication institutionnelle, les pratiques d'enseignement, ou d'interaction dans les réunions scientifiques et groupes de travail en particulier?

7 Cet article présentera les résultats de trois enquêtes. L'une d'entre elles (Etude 2), concernant le cas de l'UNI-A, a été effectuée au semestre de printemps 2016 sous forme de travail de séminaire (Cattin, 2016) dont la thématique était le plurilinguisme dans l'enseignement supérieur. Ce travail de recherche était en lien avec un projet de recherche (Etude 1) subventionné par le Fonds National Suisse ( $\mathrm{n}^{\circ} 100015147315 / 1$, 2013-2015) (Yanaprasart, 2015a). Dans la partie de discussion, nous comparerons les résultats de la recherche l'UNI A (Etudes 1 et 2) avec ceux obtenus d'une étude en cours (Etude 3) conduite à l'UNI B (Yanaprasart \& Mélo-Pfeifer, 2017) portant en partie sur la même problématique, à savoir la gestion de la diversité linguistique, les mesures de gestion et les représentations sociales des étudiants du plurilinguisme et des pratiques plurilingues dans les cours.

Figure 1 : Présentation des études

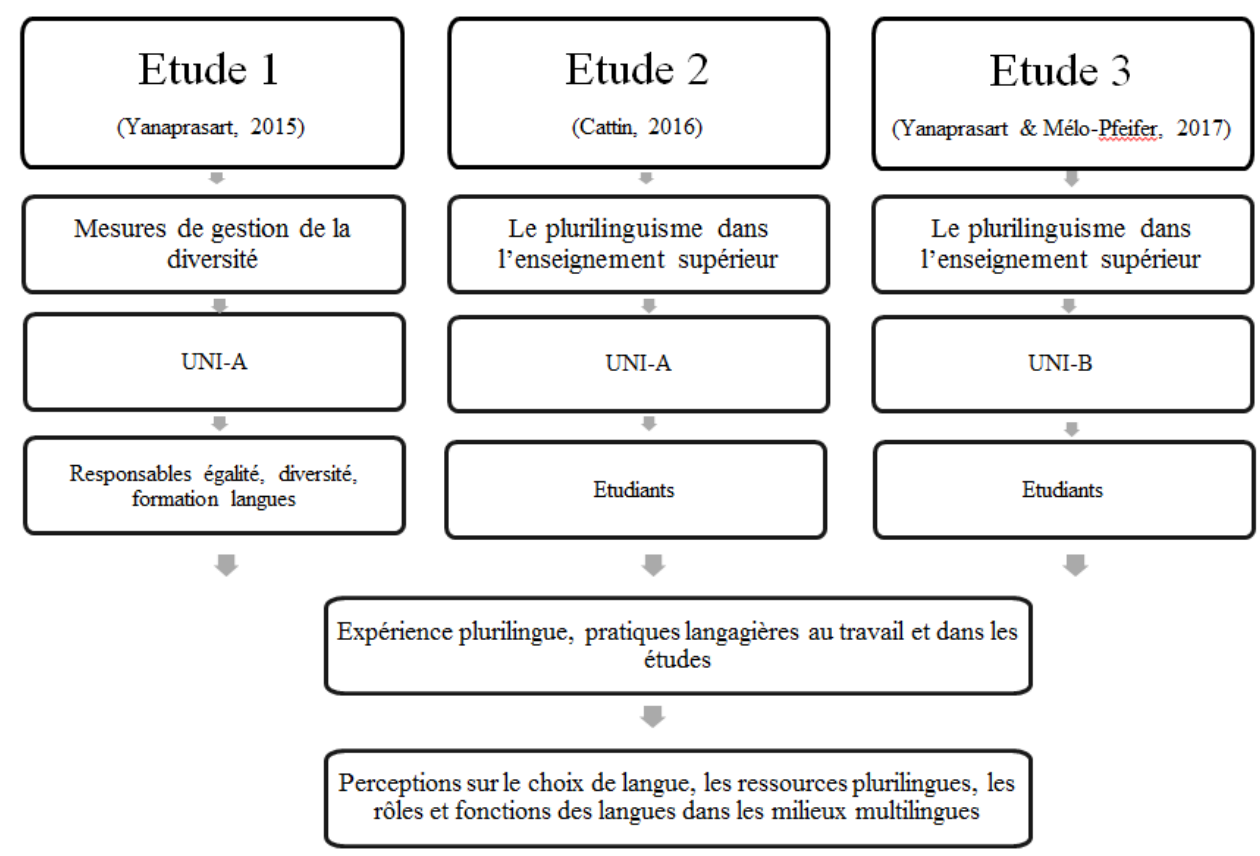

8 En partant du constat que les deux universités en Suisse Romande se sont inscrites explicitement dans le mouvement d'internationalisation de l'enseignement supérieur, nous pouvons nous demander dans quelle mesure les étudiants en font l'expérience au cours de leurs études. Cet article a pour objectif de se pencher sur la propre expérience du plurilinguisme des étudiants dans leurs études d'une part, mais aussi de questionner la représentation qu'ils ont de ce sujet, notamment de ses avantages et inconvénients en termes de pratiques dans la classe. 
9 Les trois enquêtes précitées seront analysées afin de 1) confronter la perception que les acteurs ont de l'internationalisation avec la politique linguistique institutionnelle, à savoir le parler institutionnel («Company speak ») en termes de philosophie et valeurs, le discours des « responsables de la diversité ?» («professional speak») en rapport au traitement et aux mesures, à la parole des professeurs et des étudiants en fonction des pratiques et stratégies ; 2) explorer ce que représentent les pratiques plurilingues dans le monde académique; 3) identifier une convergence ou divergence possible entre les connaissances scientifiques et la sensibilité des acteurs à ces savoirs théoriques.

La partie suivante abordera la littérature scientifique dans les domaines des défis de la diversité dans les milieux universitaires (2.1) avant de porter un regard particulier sur le choix de la langue scientifique (2.2). Les enjeux linguistiques dans le processus d'internationalisation des savoirs feront l'objet de la section 2.3 où l'accent sera mis sur le rôle de médiation du langage et des langues. Le concept de médiation sera mis en relation avec la diversité des disciplines (2.4). La section 3 traitera de l'enquête de terrain dans deux universités suisses francophones. Les résultats seront présentés et analysés dans la section 4, suivis par la discussion (section 5). L'article sera clos par la conclusion et les perspectives (section 6).

\section{Cadre théorique}

\section{Défis de la diversité dans les milieux universitaires}

11 Aujourd'hui, la diversité est promue, dans la plupart des institutions et des organisations, d'une part afin de lutter contre différentes formes de discrimination et d'autre part d'accueillir et d'intégrer les employés avec différentes valeurs et croyances. Plus important encore, la diversité est dite «bon-pour-business », tant en ce qui concerne les rendements individuels que collectifs (McMillan-Capehart, 2006 ; Marquardt et Horvath, 2001 ; Landemore, 2013).

12 Face à la globalisation économique, à l'évolution démographique de la société et au développement rapide de la technologie et de la science, à l'intensification des relations transnationales (Bruchhagen, 2008), les institutions universitaires sont amenées à se demander comment gérer et encourager la diversité académique et scientifique. Sauberer (2008) affirme que la diversité dans l'enseignement supérieur est naturellement très complexe et les dirigeants se sentent souvent incertains lorsqu'il s'agit de questions liées à la diversité. Une telle incertitude peut expliquer pourquoi de nombreuses institutions choisissent de gérer uniquement la dimension "stratégique » de la diversité. De ce fait, beaucoup d'entre elles préfèrent mettre l'accent sur l'intégration plutôt que sur d'autres éléments clés de la diversité (Cengiz 2009). Néanmoins, le document Avantages et défis de la diversité dans les milieux universitaires ${ }^{1}$ (2010) souligne clairement l'importance d'autres nombreux attributs de la diversité qui contribuent à la richesse de l'environnement pour l'enseignement et la recherche.

13 Clarke, Thomas, et Wallace (sans date) indiquent que les universités devraient non seulement encourager, mais aussi développer des formes constructives, souhaitables de la diversité en tant que stratégies commerciales légitimes pour améliorer leur prospérité à long terme. Aigare, Thomas et Koyumdzhieva (2011) précisent qu'en raison de la nature unique de ces institutions, la diversité est perçue différemment dans le contexte académique par rapport à l'environnement des affaires. Pour Meek et Wood $(1998,5)$, la 
diversité a une influence sur presque toutes les facettes d'une institution: l'accès et l'équité, les méthodes d'enseignement/apprentissage, les priorités de recherche, la gestion de la qualité, la pertinence sociale, les finances, etc. Si la réflexion sur la diversité culturelle a émergé d'une préoccupation pour ceux qui avaient été sous-représentés (Olivas, 1992) et qu'au début, les actions positives ont été largement utilisées pour obtenir l'accès à l'université pour tous (Mirela Moldoveanu, 2008), il s'avère, cependant, qu'en raison de changements en termes de "marché », la question de la diversité a cessé d'être un simple idéal social d'une entreprise classique (Barbosa et Cabral-Cardoso, 2007). Par conséquent, les universités cherchent à atteindre leurs propres objectifs, voire à améliorer leur propre performance, à maintenir la compétitivité et à gérer le rendement de la main-d'œuvre du personnel et de leurs étudiants. Pour autant, les résultats de différentes études ne sont pas concluantes ou même contradictoires (voir Aigare, Thomas et Koyumdzhieva, 2011) sur la façon dont la diversité influe sur les institutions et leur rendement.

\section{Des défis de la diversité académique au choix de la langue scientifique}

14 En ce qui a trait aux défis de la diversité mondiale, l'enseignement supérieur du $21^{\text {ème }}$ siècle se caractérise notamment par une volonté d'internationalisation, de compétitivité internationale et de globalisation (Airey, 2012). L'internationalisation est une mission primordiale de l'université qui est connue et réputée pour son adhésion à des normes mondiales, tant aux niveaux de l'internationalisation des facultés, du recrutement du personnel le plus talentueux, des formations proposées aux étudiants, qu'à ceux de l'internationalisation de l'environnement d'apprentissage, de la promotion de la mobilité universitaire, du renforcement de l'expérience internationale et de la pratique scientifique liée à la production de nouvelles connaissances. Ce processus est concrètement visible à travers le processus de Bologne, qui vise, selon Usunier (2010), à standardiser l'enseignement supérieur européen.

Sous l'étiquette 'international', synonyme de modernité et d'attractivité, l'anglais, souvent présenté comme la langue de l'internationalisation, la clé du succès (Zhu, 2014), un outil pour la pratique de l'internationalisation (Nussbaum, Moore, et Borràs, 2013), voire un moyen de communication universitaire et de socialisation qui est de plus en plus omniprésent en tant que moyen d'enseignement dans des programmes internationaux à l'échelle continentale des universités européennes (Schaller-Schwaner, 2009). A l'exemple de l'Université de Delft (Klaassen, 2008), toutes les personnes nouvellement recrutées doivent répondre aux exigences linguistiques en anglais et l'équipe scientifique doit passer des tests dans cette langue si elles ne peuvent pas fournir de certificats. A Delft, les enseignants sont contraints de suivre des cours pour améliorer leur compétence linguistique au niveau de C1. L'Université de Copenhague a introduit une certification d'enseignants comme preuve qu'ils peuvent enseigner en anglais (Cancino, 2011)

Dans la pensée populaire, l'utilisation d'une seule langue peut résoudre des problèmes de communication, accroître l'efficacité et améliorer l'égalité des chances (Kekulé, 2010). Une idéologie monolingue - l'anglais est considéré comme adapté pour les publications scientifiques et universitaires - conduit à des changements dans la politique linguistique et influe les décisions dans la mise en place des programmes d'études locaux. Pour Unterberger (2012), les processus d'internationalisation sont reflétés dans les 
programmes d'études et les efforts de marketing sont nécessaires pour se mesurer à des concurrents. Clairement, la mise en place des programmes d'études en anglais est un facteur décisif pour inclure des universités dans des classements internationaux, car de tels programmes garantissent une comparabilité internationale et augmentent le profil public de l'institution (Wilkinson, 2010).

Une étude conduite par Wächter et Maiworm (2008) portant sur les programmes enseignés en anglais dans l'enseignement supérieur européen suggère que la Suède est l'un des principaux pays dans ce mouvement vers l'usage croissant de l'anglais. Cenoz (2009) et Coleman (2006) font remarquer que l'anglais a été adopté par les académiciens espagnols comme la langue internationale de l'université. Il est perçu comme une langue dont les étudiants ont besoin pour assurer leur succès à l'université et pour leur future carrière. Selon University World News (19 novembre 2013), le nombre de cours enseignés en anglais a triplé au cours des cinq dernières années. L'objectif est double : faciliter les échanges internationaux et préparer les étudiants à la recherche. Selon Bordons et Gómez (2004), le pourcentage annuel de publications en anglais inclus dans le Science Citation Index Expanded est passé de 84,5\% en 1980 à 95,9\% en 2000.

Sur ce point, on peut identifier une opposition entre une volonté de standardisation par un recours massif à la lingua franca de l'anglais ${ }^{2}$ et à l'opposé, une culture de la diversité linguistique comme positive pour le monde académique. Cette volonté se traduit par le maintien de la diversité, considérée comme un outil décisif dans l'internationalisation de la formation et de la recherche vu le caractère multilingue et multiculturel de la société (Forster Vosicki, 2015). Autrement dit, l'anglais est nécessaire mais insuffisant.

\section{Enjeux linguistiques dans le processus d'internationalisation des savoirs : le rôle de (re-) médiation du langage et des langues}

Bien que les résultats de plusieurs études scientifiques montrent clairement la tendance du recours à l'anglais comme lingua franca en soulignant son apport à la science, les critiques qui en découlent ne portent pas sur son usage, mais sur les risques que représente un monolinguisme bien croissant dans la science et pour la science. Certes, la lingua franca internationale est indispensable pour la communication transnationale ( fonction véhiculaire), mais l'usage d'une seule langue n'est pas suffisant pour faire face ni aux divers enjeux « multilinguaculturels » (fonction identitaire) (Yanaprasart 2012, 2015b, 2016a), ni à la fabrication des savoirs (fonction constitutive) (Gajo 2013; Müller-Blaser et Pantet 2008; Steffen et Pantet 2011), ni aux défis économiques, politiques et éducatifs ( fonction sociétale) (Berthoud, Grin et Lüdi, 2013, Lüdi, Höchle Meier et Yanaprasart 2013; 2016, Yanaprasart 2016b).

Les enjeux du plurilinguisme pour la construction et la transmission des connaissances ont fait l'objet d'études, ces dernières années, dans de nombreux travaux de recherche (Castellotti et Moore (eds.), 1999 ; Berthoud, Grin et Lüdi (eds.), 2013 ; Steffen, G., 2013 ; Borg et al. (sous la direction de), 2015). L'un des résultats principaux était que les pratiques plurilingues pouvaient constituer une alternative au recours à une lingua franca unique. Certains scientifiques ont argumenté que, comme un instrument de communication rapide, l'anglais lingua franca ne devrait pas constituer LA mais L'UNE des stratégies de communication à toutes fins pratiques entre les personnes de différents répertoires. Les observations faites par plusieurs équipes du projet européen Dylan (voir Nussbaum, Moore et Borràs, 2013 ; Veronesi et al., 2013 ; Gajo et al., 2013 ; Moring et al., 
2013 ; Oltean et al., 2013) ont démontré que le choix de la langue pouvait améliorer ou masquer l'accès à la connaissance, transcender les frontières linguistiques ou réifier. L'analyse des pratiques plurilingues dans l'enseignement supérieur suggère par exemple que l'utilisation de différentes langues change notre perception de processus et d'objets, enrichit notre construction conceptuelle, résultant en un approfondissement et un raffinement de compréhension conceptuelle (Lüdi, 2015). Comme l'ont souligné MüllerBlaser et Pantet (2008), le fait que les étudiants sont obligés de prêter attention aux détails de la deuxième langue pour comprendre le contenu amène à examiner de plus près les mots et approfondit leur réflexion conceptuelle dans les langues utilisées. L'étude de Yanaprasart et Lüdi (2017) approuve cet avis : des contenus conceptuels sont vus sous différents angles, à travers le parler plurilingue.

Dans cet ordre d'idées, Forster Vosicki (2015 : 285) rejoint Usunier (2010)) en écrivant que « les contenus scientifiques n'existent pas de manière autonome en-dehors du langage. Les langues ont un rôle configurant dans la construction et le transfert du savoir.» En effet, la réflexion sur la place de la langue d'apprentissage et d'enseignement dans l'université pose tout à la fois deux questions importantes. La première interroge les conditions de la pratique de la langue. La seconde question s'intéresse au rôle et à la place de la langue et au langage dans le processus complexe de la construction et de la transmission du savoir scientifique. Les réflexions de Berthoud (2016), s'appuient sur la reconnaissance du langage comme instrument de médiation, ce en se référant au sens donné par Vygostski (1997): "le langage fonctionne comme principal système de médiation pour la pensée, médiation symbolique » (cité dans Berthoud, 2016: 169). Les savoirs n'existent qu'à travers des supports élaborés et reconnus socialement (Berthoud et Gajo, 2016). D'autre part, le langage et les langues fonctionnent comme instruments de négociation et de contrôle de notre rapport au monde dans le processus de médiation (activité discursive et méta-discursive) : languaging (Berthoud, 2016).

La re-médiation (ou double médiation) intervient au moment où le discours est formulé dans une autre langue que la langue première. Dans ce processus de formulation, la langue peut intervenir fondamentalement dans la construction des connaissances (Escudé et Gajo, 2016). Cette deuxième médiation permettra à la fois d'ajuster plus explicitement les moyens linguistiques disponibles et d'amener un regard alternatif sur les liens entre discours et savoirs, selon Gajo (2013: 99) : «C'est à ce double niveau que le plurilinguisme sert à la fois de loupe et de ressource supplémentaire dans le processus des savoirs ». Berthoud (2016) évoque deux fonctions de la langue : la fonction véhiculaire (la langue sert de transmetteur pour une diffusion d'information et optimiser une communication efficace, rapide et économique (outil communicatif) - l'optimisation) et la fonction constitutive en augmentant les outils de conceptualisation (médiation langagière et métalinguistique des savoirs (instrument cognitif) - le rôle de révélateur de la médiation langagière des savoirs). Dans cette optique, les pratiques langagières interviennent de manière structurante sur les savoirs à double fonction: schémas cognitifs et modèles d'action. Le plurilinguisme est en quelque sorte un choc « renforçateur » entre plusieurs façons de penser et d'agir pour augmenter la conceptualisation et pour optimiser la communication (instrument interactionnel et stratégique). 


\section{Médiation et disciplines dans leur diversité}

23 Ces arguments en faveur du plurilinguisme doivent toutefois être ramenés à la réalité du monde académique actuel tel que le vivent probablement la plupart de ses membres: l'anglais y occupe une place prépondérante, qui risque de devenir hégémonique selon Hamel (2013), surtout dans les sciences exactes. L'idéal plurilingue que promeut notamment le système de Bologne se heurte ainsi à des pratiques parfois fortement monolingues. Suivant la précision d'Usinier (2010), ce risque concerne moins les sciences exactes que d'autres telles que les sciences humaines, en raison d'un rapport au langage différent. Il est, en effet, davantage constitutif des problématiques abordées par les sciences humaines que celles des sciences exactes. D'après Berthoud et Gajo (2016), les différentes disciplines ont un rapport différent avec le langage, les langues et les discours, selon la nature des disciplines et le type d'activité scientifique. Dans les sciences de la matière et de la nature, l'anglais est aujourd'hui bien établi comme moyen de communication et de publication, vu que la langue est un moyen d'expression parmi d'autres (images, diagrammes, tableaux, formules, chiffres, etc.) et que les objets d'étude dans ce domaine sont traités dans des contextes standardisés. En sciences économiques, l'anglais est devenu la langue des affaires et de l'économie mondiale, tandis qu'en sciences sociales, l'anglais représente tout à la fois un atout et un problème. Cela dit, la langue reste un moyen de transmission des connaissances incontournable car les pratiques sociales sont étroitement liées aux pratiques linguistiques. Quant aux sciences juridiques, l'anglais constitue tout à la fois une opportunité et un risque. Des affrontements entre différentes pratiques institutionnelles et juridiques dans des décisions constitutionnelles sont indispensables, d'où des difficultés lors de l'interprétation de textes et de normes. Pour finir, la domination croissante de l'anglais semble poser le plus de problème au domaine des sciences humaines où la production scientifique est inscrite dans la langue. L'usage de l'anglais risque de standardiser les concepts, les savoirs et les discours produits et transmis.

Lorsque l'on parle des pratiques scientifiques aussi bien pour les disciplines « dures » que pour les disciplines "molles ", le rôle de médiation de la langue varie d'une activité à l'autre. S'il est clair que la langue exerce principalement sa fonction constitutive dans le processus de fabrication des savoirs, c'est surtout dans la circulation spécialisée que le lien avec la fonction véhiculaire de la langue occupe la place centrale. Le cas de la diffusion large est dans une étape intermédiaire. En la mettant en relation avec les notions de médiation et de re-médiation évoquées précédemment, la fonction constitutive dans la fabrication des savoirs est clairement la fonction de médiation où les savoirs sont en-formation (l'en-formation : la fabrication des savoirs dans un processus de travail d'équipe). Dans une diffusion spécialisée ou ciblée (information dans un événement scientifique), c'est la fonction de remédiation qui exige un ajustement des moyens linguistiques pour assurer une communication transparente. La diffusion plus large des savoirs s'inscrit dans le travail de re-médiation. Il s'agit non seulement de diffuser des connaissances, mais aussi de les adapter aux besoins d'un public qui ne possède pas le même degré d'expertise, le même savoir disciplinaire ou la même culture scientifique. C'est dans cette étape que se situe l'enjeu du travail de vulgarisation qui prime la formation mettant en évidence le lien entre science et communauté (voir Berthoud 2016 ; Berthoud et Gajo, 2016 pour plus de détails). 

sur la diversité des langues afin de maintenir une pluralité de perspectives bénéfique à la science. Partant de ce fait, la partie suivante présentera nos trois études des représentations sociales des acteurs dans les milieux académiques à l'égard de la politique linguistique prise entre une tendance à l'internationalisation traduite par la standardisation linguistique et une valorisation des ressources plurilingues individuelles au service de la qualité des savoirs. Nous commencerons par présenter la méthodologie des trois travaux dans la section suivante.

\section{Enquête de terrain dans deux universités suisses francophones}

Les deux méthodes principales utilisées concernent l'usage de questionnaire semi-ouvert (auprès de quinze étudiants à l'UNI-A (Cattin, 2016) et de trente-et-un étudiants à l'UNI-B (enquête en cours Yanaprasart et Mélo-Pfeifer, 2017), ainsi que l'analyse d'entretiens semi-directifs (auprès de la responsable adjointe de la diversité, de la directrice du Centre de langue et de la directrice du bureau de l'égalité à l'UNI-A (Yanaprasart, 2015a; Yanaprasart et Lüdi, 2017). La langue des questionnaires et des entretiens est le français, la langue locale et d'enseignement des deux universités.

Dans le cadre de l'UNI-A (Cattin, 2016), un questionnaire a été soumis à quinze étudiants. Le but était d'obtenir des réponses d'étudiants provenant de facultés variées, mais d'avoir tout de même plusieurs réponses par faculté pour donner une certaine représentativité (bien que limitée) à l'échantillon, particulièrement pour la faculté des lettres qui offre une forte variété de domaines d'études. La récolte de données a été effectuée dans les couloirs et cafétérias de trois bâtiments de l'université, ciblant ainsi des étudiants inscrits en lettres, en sciences sociales et politiques et en Haute école de commerce.

Le questionnaire porte d'abord sur l'expérience personnelle que les répondants ont du plurilinguisme dans leurs études. Il peut par exemple s'agir d'éventuels cours donnés dans d'autres langues que le français à l'université. Des questions plus ouvertes seront ensuite posées au sujet de l'emploi de l'anglais et du plurilinguisme dans la science en général et ce qu'en pensent personnellement les répondants. Les questions sont formulées de façon à avoir accès à des réponses spontanées. Les thèmes mentionnés dans la partie théorique sont ainsi questionnés de la manière la plus neutre possible. Le but est de découvrir la position spontanée et actuelle des étudiants sur ces questions.

Le profil des répondants de l'UNI-A est le suivant: sept sur quinze, d'un âge moyen de 21,2 ans, sont de sexe féminin. Deux personnes se sont inscrites en master (à la Faculté de la Haute école de commerce - HEC) et treize en bachelor ( 5 en lettres, dont 2 en filière langue ; 3 en sciences sociales et politiques, dont 1 en filière langue ; 2 en HEC, 2 en droit et 1 en géographie). Pour ce qui est de leur profil linguistique, tous sont au minimum bilingues français-anglais. Dix d'entre elles parlent en plus l'allemand alors que deux répondants connaissent le suisse allemand. L'italien est parlé par sept étudiants, l'espagnol par quatre personnes interrogées. L'arabe est la langue première de deux étudiants, un répondant possédant le portugais comme langue première.

Quant au profil des étudiants de l'UNI-B enquêtés (Yanaprasart et Mélo-Pfeifer, 2017), douze personnes sont des étudiants inscrits en diplôme (DEFLE), deux en bachelor (BAFLE) et dix-sept en master en en français langue étrangère (MAFLE). 22,5\% sont de 
sexe masculin. Les interrogés sont âgés entre 20 et 42 ans. Sur le plan linguistique, six interrogés sont francophones, trois russophones, deux italophones, deux hispanophones, un anglophone, un germanophone, un lusophone, un sinophone, une personne parlant le persan, un bulgarophone, un lituanophone, un roumanophone. De plus, 25,8\% (huit personnes) ont deux langues premières (roumain/russe (2), français/albanais, chinois/ anglais, turc/italien, italien/sicilien, français/japonais, russe/espagnol). Les autres langues parlées sont l'anglais (26 personnes), le français (21), l'allemand (6), l'espagnol (4), le portugais, l'arabe et le chinois parlés par deux personnes, le japonais, le danois, l'italien, le bulgare, le catalan, l'ukrainien, l'ilocano, le latin, le russe, le tigrinya, l'azeri, l'uzbek, le tazath, le turc, le kirghize font partie des répertoires linguistiques des étudiants sondés. A la question "si vous vous considérez comme plurilingue ", tous y répondent affirmativement. Quatre personnes $(12,9 \%)$ se disent bilingues. La majorité est trilingue $(35,4 \%) ; 19,3 \%$ parlent quatre langues, $12 \% 9$ peuvent s'exprimer en cinq langues et six langues sont parlées par le même pourcentage, une personne parle sept langues et une autre huit langues.

\section{Résultats}

\section{La politique linguistique de l'UNI-A et sa gestion de la diversité linguistique}

31 Comme toutes les universités suisses ${ }^{3}$, l'UNI-A a son propre bureau de l'égalité des chances pour promouvoir la diversité de ses membres. Elle donne trois arguments pour promouvoir et gérer adéquatement la diversité : une culture d'ouverture, l'expérience unique de chacun et l'inclusion de la réussite et de la compétitivité. Comme il est écrit dans son plan d'intentions ${ }^{4}$, "les expériences différentes desmembres de la communauté universitaire sont souvent à l'origine de l'innovation scientifique et du développement de l'Université en tant qu'organisation». En 2012, une responsable adjointe dont la tâche était de promouvoir la diversité a été nommée.

En matière de langue, l'UNI-A s'est dotée en 2003 (jusqu'en 2011) d'une Commission de politique linguistique afin de concevoir une politique harmonisée au niveau institutionnel. Ses tâches principales, en début de sa mission, étaient de fournir « une politique linguistique plurilingue" et d'édicter des objectifs pour s'adapter à l'internationalisation de l'enseignement supérieur en fournissant des arguments dans ce sens. Un document intitulé Le rôle du plurilinguisme dans la stratégie d'internationalisation de l'UNI-A a identifié les différents objectifs de la Stratégie d'internationalisation de l'Université en mettant au clair que le profil plurilingue doit être partie intégrante de la formation des étudiants.

Dans le règlement adopté en 2007, il est stipulé que la langue officielle est le français. Des cours peuvent cependant être donnés dans d'autres langues pour autant que l'enseignant en ait une maîtrise suffisante (fixée au niveau B2 ou C1 $1^{5}$ ). Par ailleurs, les étudiants doivent être informés des éventuels prérequis et des offres de formation dans ce sens doivent être disponibles. Le plurilinguisme au niveau de la langue d'enseignement des cours est surtout encouragé au niveau du master, tandis que le français est la langue d'enseignement pour le niveau du bachelor. 

laboratoire Doplab en 2013, développée dans deux grandes institutions lausannoises, est installée dans les salles de classe pour encourager les interactions et la participation en particulier dans les grands auditoires. Les étudiants peuvent poser des questions de façon anonyme à partir de leurs téléphones intelligents. A cet égard, les pratiques plurilingues sont encouragées d'une manière positive, selon la responsable adjointe de la diversité : «si les étudiants ne sont pas très sûrs de leur anglais, même si la langue d'enseignement est l'anglais, ils peuvent poser des questions également en français et l'enseignant peut répondre en anglais. Et pour des étudiants non francophones, s'ils veulent écrire en français, des erreurs d'orthographe sont tolérées. C'est là où la langue peut jouer un rôle dans l'inclusion ». Néanmoins, telle que présentée par la directrice responsable en formation linguistique, «un certain nombre d'enseignants ne font pas assez pour exploiter les ressources linguistiques de leurs étudiants. Ils restent dans une perspective monolingue de l'enseignement et de la communication ».

\section{Représentations sociales}

Le concept de représentation est utilisé par Durkheim (1960) pour expliquer qu'entre les divers groupes sociaux circulent des représentations sur les autres. Boyer (1995) met cette notion en relation avec les aspects « ethnosocioculturels» de chaque groupe. On 
trouve l'adjectif «social» dans la définition de Jodelet (2003: 53) pour qui la représentation sociale est «(...) une forme de connaissance, socialement élaborée et partagée, ayant une visée pratique et concourant à la construction d'une réalité commune à un ensemble social ». Ce concept a donc une importance particulière dans la vie en société, notamment dans le domaine de l'assimilation de la connaissance où il joue un rôle constitutif. L'auteure la distingue notamment de la science: il peut y avoir une divergence entre deux représentations d'un même objet, une construite par le biais de la science et une autre par celui du sens commun par exemple. Cette notion est centrale dans nos enquêtes qui ont pour objectif de confronter les principaux avantages du plurilinguisme dans l'enseignement supérieur du point de vue de la science et tel que se le représentent les étudiants.

\section{Représentations sociales des étudiants à l'égard des pratiques plurilingues dans leurs études}

Il s'avère que près de la moitié des personnes interrogées (sept sur quinze) suivent des cours donnés dans une autre langue que le français dans le cadre de leurs études à l'UNIA. Sur ces sept, trois personnes le font en raison d'une inscription dans une filière «langues». Deux répondants inscrits en master font également partie de ces sept personnes. La langue d'enseignement principalement mentionnée est l'anglais (dans six des sept cas), ainsi que l'allemand, l'italien et l'espagnol (qui n'apparaissent qu'une fois). Une seule personne précise que le fait de devoir suivre des cours dans une autre langue lui pose des problèmes, mais sans les détailler.

Ces résultats reflètent bien la politique officielle de l'UNI-A en matière de langue d'enseignement : en effet, sur les sept personnes suivant un cours donné dans une autre langue que le français, trois le font dans le cadre particulier d'une filière de langues. Restent quatre personnes, dont deux sont en master et deux qui suivent un cursus de bachelor qui ne doit pas nécessairement être plurilingue au niveau du règlement de l'UNI-A.

41 Le fait qu'une seule personne mentionne avoir un problème (sans le préciser) avec un enseignement dans une autre langue que le français ne permet pas d'identifier une disposition négative vis-à-vis de ce type d'enseignement de la part des étudiants. Leur disposition semble plutôt être positive, même s'il faut rester prudent sur ce point en raison de la faible taille de l'échantillon et de l'absence de commentaires plus approfondis.

\section{Représentations sociales des étudiants à l'égard du plurilinguisme de leurs enseignants}

Un autre volet du plurilinguisme à l'UNI-A interrogé concerne les ressources plurilingues des professeurs. Trois personnes disent ne pas y voir d'avantages et huit sont sans avis sur ce point.

Treize répondants suivent un cours donné par un professeur qui n'y utilise pas sa langue première. Cela pose un problème à $38,5 \%$ des répondants en mettant en avant des problèmes de compréhension dus à la compétence linguistique du professeur, plus particulièrement à son accent ou sa prononciation. Ces problèmes de compréhension liés à la compétence linguistique du professeur indiquent qu'il y a peut-être un problème 
linguistique, qui n'est d'ailleurs que peu contrôlé au niveau du recrutement. Le niveau $\mathrm{B} 2 / \mathrm{C} 1$ requis est ainsi purement indicatif et aucun examen n'est effectué pour le vérifier.

$26,5 \%$ de l'échantillon voient au contraire des avantages à une telle situation : ils citent surtout l'apport de perspectives différentes que permet une telle situation (23\%), ainsi qu'une certaine pratique d'autres langues (7,6\%). Le fait que «l'apport de perspectives différentes » soit cité trois fois comme un avantage semble positif car il confirme qu'un enseignement sur un modèle plurilingue permet un approfondissement des concepts, bien ressenti concrètement par les étudiants concernés.

A la question portant sur la lecture régulière d'articles majoritairement écrits en anglais, quatre personnes ont répondu avoir des problèmes de compréhension (dus à une compétence insuffisante). Les onze autres (dont un locuteur natif de l'anglais) n'ont pas de problème avec ceci. Même si tous les répondants disent avoir des compétences en anglais, environ 26,5\% estiment ne pas en avoir assez pour les lectures demandées à l'université. Si l'on se tient à la formule de l'anglais comme « langue nécessaire mais non suffisante ", il y aurait un effort de formation à faire dans cette langue. Des offres de formation en langues existent à l'UNI-A, ce qui tendrait à attribuer la responsabilité de ce problème aux étudiants eux-mêmes.

Représentations sociales des étudiants à l'égard de leur propre plurilinguisme

A la question portant sur la mobilisation d'autres langues que la langue première dans les études, tous les répondants ont cité au moins un point positif, et huit au moins un négatif. Les réponses (le chiffre entre parenthèse indique le nombre de fois que cette réponse est apparue) sont résumables comme suit :

Tableau 1 : Réponses sur la mobilisation des langues

\begin{tabular}{|l|l|}
\hline Points positifs : & Points négatifs : \\
\hline $\begin{array}{l}\text { Permet de pratiquer la langue (développement et } \\
\text { entretien des compétences) (10) }\end{array}$ & $\begin{array}{l}\text { Problèmes d'équivalence des termes entre } \\
\text { les langues (4) }\end{array}$ \\
\hline Amène une diversité de points de vue (4) & $\begin{array}{l}\text { Importants efforts d'apprentissage à } \\
\text { fournir, fatigue liée à cela (4) }\end{array}$ \\
\hline Accès à davantage de sources d'information (4) & Erreurs de compréhension (1) \\
\hline Atout pour la communication (2) & \\
\hline $\begin{array}{l}\text { Permet de mieux comprendre certaines nuances de } \\
\text { sens (1) }\end{array}$ & \\
\hline Permet de connaître de nouvelles personnes (1) & \\
\hline
\end{tabular}

Les avantages cognitifs d'un apprentissage plurilingue tels qu'ils ont été montrés dans la littérature (Moore, 2006 ; Lüdi et Py, 2003 ; Cenoz, 1997) se retrouvent dans les réponses des étudiants. Ce type d'avantage n'est toutefois cité que relativement peu de fois en comparaison avec celui, plus direct, de la pratique de la langue (cité dix fois). L'avantage consistant en une meilleure assimilation de connaissances n'est ainsi cité qu'une seule 
fois, tandis que la plus grande créativité des personnes plurilingues n'est pas du tout citée. Même si les réponses à cette question sont globalement positives (il y a plus de points positifs que négatifs), les étudiants ne semblent pas tous conscients de tous les avantages qu'apporte un apprentissage plurilingue.

De plus, ce sont surtout les répondants inscrits en lettres qui ont cité comme points positifs ce que l'on peut rapprocher des "avantages cognitifs» soulignés par des scientifiques (Grosjean, 2013 ; Bialystok, 1999 ; Cook 1997 ; Hakuta \& Diaz, 1985 ; Pearl \& Lambert, 1962). En effet, quatre étudiants en lettres (sur cinq) ainsi qu'un en droit et un en SSP ont cité la diversité des points de vue et une meilleure compréhension de certaines nuances de sens comme avantage. Bien qu'il soit délicat d'identifier une corrélation entre l'appartenance à une faculté et le type de réponse à cette question sur la base d'aussi peu de réponses, cette étude pourrait constituer un point de départ, voire une piste à explorer plus en détail. Il est d'ailleurs relativement intuitif d'affirmer que les étudiants en lettres sont davantage sensibles à ce genre de problématique que d'autres. A ce sujet, l'étude d'Airey (2011) montre que si les étudiants suédois ont pensé que la langue d'enseignement était sans importance et que quelle que soit la langue d'enseignement (le suédois ou l'anglais) n'avait aucune influence sur leurs perceptions du sujet en physique, c'est parce qu'ils étaient ignorants d'un certain nombre de problèmes apparus lors de l'enseignement de la physique en anglais et qu'ils ont possédé une compétence restreinte pour décrire efficacement des concepts disciplinaires en anglais, interagir avec succès dans les lectures, écouter et prendre des notes simultanément - de tels problèmes pourraient entraver l'apprentissage. En parallèle, les enseignants en tant qu'experts d'un contenu sont généralement inconscients de ce que l'aspect de l'apprentissage linguistique implique pour leur enseignement (Smit, 2003)

La dernière question porte sur l'impact de l'usage de l'anglais lingua franca dans la science en général. Tous les répondants ont cité au moins un point positif et onze au moins un point négatif. Si la formulation des réponses peut varier d'un répondant à l'autre, on peut tout de même les regrouper dans des thématiques communes.

Tableau 2 : Réponse sur l'usage de l'anglais

\begin{tabular}{|l|l|}
\hline Points positifs : & Points négatifs : \\
\hline $\begin{array}{l}\text { Recours à une langue véhiculaire, } \\
\text { universalité (14) }\end{array}$ & $\begin{array}{l}\text { Implique des efforts supplémentaires d'apprentissage et de } \\
\text { lecture (fatigue et restriction d'accès) (9) }\end{array}$ \\
\hline $\begin{array}{l}\text { Accès pour plus de personnes à la } \\
\text { littérature (7) }\end{array}$ & Manque de diversité (sans plus de précision) (2) \\
\hline $\begin{array}{l}\text { Choix plus vaste à disposition } \\
\text { dans la littérature (5) }\end{array}$ & Perte de précision sur certains détails (2) \\
\hline $\begin{array}{l}\text { Homogénéité } \\
\text { nomenclature (3) dans }\end{array}$ & Perte d'identité (personnelle ou nationale) (1) \\
\hline $\begin{array}{l}\text { L'anglais est une langue «facile » } \\
\text { (1) }\end{array}$ & \\
\hline
\end{tabular}




\section{Les points négatifs portent surtout sur les efforts supplémentaires qu'implique le recours à l'anglais, tant au niveau individuel qu'au niveau global. Ce type d'avantage et d'inconvénient est donc surtout lié à l'usage direct de la langue plutôt qu'à ses conséquences profondes. Le fait que des points négatifs soient cités moins de fois que ceux positifs (et que quatre personnes n'aient mentionné aucun point négatif) attire particulièrement notre attention: une interprétation possible serait que l'usage de l'anglais comme la langue de la science n'a pas été particulièrement perçu comme une menace par les étudiants interrogés.}

\section{Discussion}

51 L'objectif de ces enquêtes était d'une part d'avoir une meilleure compréhension de la gestion de la diversité en matière de politique linguistique et des mesures pour promouvoir le plurilinguisme dans les milieux académiques, ce à l'exemple d'une université suisse francophone l'UNIL-A et, d'autre part, d'explorer les représentations que ses étudiants ont du plurilinguisme dans le monde académique, ainsi que de confronter leur expérience dans ce domaine à la politique linguistique officielle de l'institution.

Sur ce point, il semble que les recommandations de la Commission de politique linguistique se reflètent fidèlement dans les résultats à plusieurs niveaux, à savoir le nombre de langues maîtrisées par les étudiants et l'enseignement plurilingue qui existe dans leur cursus. Ajouté à cela, des professeurs avec leur provenance variée apportent des avantages en termes d'apprentissage. Des problèmes liés à la lingua academica (l'anglais comme les autres langues) sont toutefois soulevés: la compétence en anglais est insuffisante chez $26,5 \%$ des répondants et la compétence linguistique de professeurs enseignant dans une autre langue que leur langue première laisse à désirer dans $38,5 \%$ des cas.

53 Il ressort, par ailleurs, des résultats que les étudiants interrogés ont globalement bien conscience des avantages et inconvénients de l'utilisation de l'anglais dans la science et $\mathrm{du}$ caractère plurilingue des études universitaires. Cependant, certaines réponses n'apparaissent que très peu de fois, ce qui laisse penser qu'il y a bien un décalage entre les avantages du plurilinguisme tels que se le représentent les étudiants et les avantages avancés par la littérature scientifique tant citée par l'observatoire européen du plurilinguisme ${ }^{6}$. Les étudiants en lettres semblent d'ailleurs avoir une plus grande sensibilité à cette problématique car ils ont mentionné plus d'avantages par rapport aux étudiants d'autres disciplines. L'hypothèse serait que l'appartenance à une faculté aurait une influence sur les réponses des étudiants : ceux inscrits en lettres pourraient ainsi être davantage sensibles aux avantages du plurilinguisme que les autres. Il est possible que les étudiants en lettres perçoivent davantage le rôle constitutif de la langue, alors que pour les autres disciplines, la langue tend à jouer le rôle essentiellement véhiculaire.

54

Il s'agit toutefois de la seule «corrélation » qui a pu être établie entre l'appartenance facultaire et une réponse. Cette variable ne semble donc jouer qu'un rôle mineur dans l'échantillon sélectionné. Ceci amène aux nécessaires limites de cette recherche. L'échantillon limité à quinze répondants n'est bien sûr pas représentatif des facultés qui y sont incluses, mais offre plutôt des pistes de réflexion. La distribution des répondants

Recherches en didactique des langues et des cultures, 15-2 | 2018 
selon les facultés permet ainsi d'avoir une diversité de profils, mais ne permet toutefois pas d'en tirer des corrélations fiables. On peut cependant imaginer un échantillon plus varié, en interrogeant des étudiants en sciences exactes par exemple.

Par ailleurs, bien que l'enquête par questionnaire ait pour but de récolter des informations claires et précises, le fait qu'il soit mené auprès d'une population limitée ne nous a pas permis d'approfondir tant quantitativement que qualitativement les réponses aux questions semi-fermées. Afin de compenser ce biais, l'étape suivante vise à mener des entretiens semi-directifs ont été prévus (Yanaprasart et Mélo-Pfeifer, 2017, enquête en cours) pour mieux connaître la notion de plurilinguisme et sa place dans l'enseignement supérieur. Bien que cette étude soit en cours, nous voulons comparer ici les résultats de l'enquête l'UNI-A avec les résultats intermédiaires de l'UNI-B dont le public interrogé concerne les étudiants en didactique du français langue étrangère. Trente-et-un étudiants ont rempli le questionnaire dont les questions portent sur les pratiques plurilingues dans les cours et la mobilisation des compétences plurilingues dans leur apprentissage, en termes d'atouts-avantages et d'obstacles-inconvénients.

Nous pourrons classer leurs réponses comme suit.

Tableau 3 : Pratiques plurilingues et mobilisation des compétences plurilingues

\begin{tabular}{|c|c|}
\hline Avantages & Inconvénients \\
\hline $\begin{array}{l}\text { "Atouts cognitifs" } \\
\text { Ouverture d'esprit plus grande; Meilleure } \\
\text { compréhension du monde; avoir différentes } \\
\text { perspectives; Richesse culturelle/intellectuelle; } \\
\text { Développement cognitif; Accroissement de } \\
\text { l'élasticité d'esprit; Mobiliser des logiques, des } \\
\text { tournures de pensée différentes; Voir les } \\
\text { différentes perspectives; Un enrichissement. }\end{array}$ & \\
\hline $\begin{array}{l}\text { "Atouts communicatifs" } \\
\text { Grand avantage dans la communication; Mieux } \\
\text { se faire comprendre; Clarification des savoirs, } \\
\text { pouvoir exprimer dans la langue la mieux parlée; } \\
\text { mieux transmettre nos idées; Accès à plus } \\
\text { d'informations; Plus de précision donc facilité de } \\
\text { compréhension; Développer une compréhension } \\
\text { passive. }\end{array}$ & $\begin{array}{l}\text { "Obstacles communicatifs" } \\
\text { Niveau de langue des différents } \\
\text { intervenants; Difficulté/Problème de } \\
\text { compréhension (11); Certains mots/ } \\
\text { concepts intraduisibles; problème de } \\
\text { mélange de langues; Difficulté à s'exprimer, } \\
\text { donner son avis, oser parler dans une } \\
\text { langue partiellement maîtrisée, arriver à } \\
\text { exprimer son idée clairement (5); Réussir à } \\
\text { transmettre des idées avec le même degré } \\
\text { de complexité ou avec autant de charge } \\
\text { émotionnelle que dans la langue } \\
\text { maternelle; Peur de ne pas bien parler. }\end{array}$ \\
\hline $\begin{array}{l}\text { "Atouts professionnels et relationnels" } \\
\text { Une valeur sur le marché du travail ; Entrer en } \\
\text { contact avec d'autres communautés. }\end{array}$ & $\begin{array}{l}\text { «Problème d'identité » } \\
\text { Reconnu comme étranger }\end{array}$ \\
\hline
\end{tabular}




\begin{tabular}{|c|c|}
\hline $\begin{array}{l}\text { "Atouts d'apprentissage" } \\
\text { Environnement d'apprentissage; développer les } \\
\text { connaissances linguistiques; Développer le } \\
\text { plurilinguisme; s'habituer à employer plusieurs } \\
\text { langues; une porte ouverte à l'apprentissage des } \\
\text { autres langues, pouvoir comparer les structures } \\
\text { de plusieurs langues et mieux les comprendre; } \\
\text { Apprentissage de nouveaux mots; Amélioration } \\
\text { des compétences linguistiques; Regard sur sa } \\
\text { propre langue/comparaison; Amélioration des } \\
\text { connaissances en plusieurs langues; Création des } \\
\text { liens/connexions entre les langues; Lecture de } \\
\text { textes originaux. }\end{array}$ & $\begin{array}{l}\text { "Défis d'apprentissage" } \\
\text { Fatigue, Plus de temps et d'effort à } \\
\text { fournir (3); Ralentissement; Sentiment de } \\
\text { frustration; Lent avancement (lecture, } \\
\text { écriture); Impression de non-progrès ou } \\
\text { d'être exclu ; Maîtrise du langage spécifique } \\
\text { (connaissance); Risque de confusion; } \\
\text { Etudes plus compliquées. }\end{array}$ \\
\hline & Il n'y en a pas (2) \\
\hline
\end{tabular}

57 Sur la base de ces résultats, nous pouvons constater qu'en termes d'avantages, ceux-ci sont souvent liés aux atouts aussi bien cognitifs que communicatifs. Le problème d'identité (fonction identitaire) est évoqué par une personne, de même qu'une réponse s'appuie sur l'avantage professionnel. De nombreux répondants disent apprécier l'environnement d'apprentissage plurilingue dans lequel les participants peuvent développer leurs compétences linguistiques en différentes/plusieurs langues d'une manière individuelle et collective, tant activement que passivement. En ce qui concerne les inconvénients, il s'agit principalement de problème de compréhension cité par 47,8\%, à cause de leur compétence insuffisante ou du bas niveau de langue des enseignants. $21,7 \%$ des répondants soulignent une difficulté dans l'expression. Deux personnes ne voient aucun inconvénient concernant les pratiques plurilingues dans les cours, alors qu'un répondant a fait le lien entre mal parler et le statut d'étranger, ce qui pourrait avoir un effet négatif sur le plan émotionnel. Pour finir, l'aspect cognitif n'a aucunement été mis en question. Une hypothèse serait que la revalorisation du plurilinguisme soit d'abord fondée sur l'avantage cognitif de personnes bi-plurilingues (Furlong, 2009).

\section{Conclusion}

Les résultats de nos trois enquêtes ont illustré d'une part une action auprès des universités et se sont attachés d'autre part à la perception des étudiants par rapport à la place et la signification du plurilinguisme dans leurs études. On peut constater qu'un profil plurilingue est nécessaire pour les étudiants et ceux-ci sont conscients que chaque langue est une ressource aussi bien pour eux que pour la société. A la question « Si ils se considèrent comme plurilingues", l'ensemble des répondants ont répondu affirmativement partant du principe qu'une variété de profils composés de langues différentes et à des niveaux de compétence multiples ne pose pas d'obstacle majeur à l'apprentissage dans un environnement académique favorisant une compétence plurilingue transversale et que la dimension multilingue a un but inclusif et intégratif. Il s'agit d'une conception plurilingue et non monolingue du plurilinguisme.

S'il est souvent présupposé que l'internationalisation signifie l'usage de l'anglais, l'internationalisation, à notre sens, concerne la relation entre plusieurs cultures et 
langues dans la même institution éducative et la possibilité de pratiquer la diversité en tant que ressource dans l'éducation. L'idée que la diversité linguistique et culturelle serait effectivement un atout dans l'enseignement supérieur transnational se situe au cœur de cet article. Une telle diversité pourrait permettre à des étudiants d'identifier des questions liées aux thèmes internationaux dans le contexte de leur champ d'étude et leur donner la possibilité de percevoir eux-mêmes en tant que citoyens globaux, bien que des problèmes, tensions et conflits souvent en résultent aussi.

La langue d'enseignement/apprentissage est loin d'être un outil neutre. Cependant, l'internationalisation des universités ne devrait pas aborder en termes simplistes de la polarité traditionnelle entre les pratiques locales et l'usage des langues nationales d'une part et l'invasion d'une lingua franca internationale, l'anglais, pour accommoder l'influx des enseignants et étudiants en mobilité transnationale d'autre part. En réalité, l'internationalisation des universités représente une nouvelle hybridité culturelle et linguistique basée sur une interdépendance culturelle, avec le potentiel de développer de nouvelles formes d'identités libérées d'une pensée binaire traditionnelle "nous-et-eux » (Pierce, 2003), un nouvel esprit d'ouverture à l'égard des rôles de soi et des autres, résultant en nouveaux modèles de pratiques communicatives.

Dans un monde d'éducation globalisé où la standardisation et la normalisation ont pour but de minimiser la diversité (Preisler et al., 2011), voire œuvrer pour l'uniformité (Unterberger, 2012), l'intégration de l'anglais lingua academica dans les programmes d'études, à côté des langues locales, fera son sens, avant tout, lorsque ces langues garantiront un accès équilibré et complémentaire aux savoirs scientifiques dans leur fabrication, diffusion et vulgarisation. Dans cet objectif, ce sera ensemble et non d'une façon séparée que la diversité des langues peuvent jouer un rôle de complémentarité et d'intégration pour une communication efficace, rapide et économique tout en gardant la complexité, l'équité et l'originalité du message. C'est surtout dans le «troublesome space » (Montgomery, 2011) transformatif (Savin-Baden, 2008) que l'usage d'une langue peut se faire non en tant que code simple, fixé et rigide, mais comme un outil de « translanguaging " (García et Wei, 2014), certes complexe, mais flexible, dynamique et évolutif afin de mieux explorer les savoirs en-formation au service de leur qualité. Une diversification et une "standardisation épaisse" (Usunier, 2013) des savoirs seront optimales dans un environnement où les cultures et langues différentes ont l'occasion de s'interagir, ce dans des perspectives hybrides et multilatérales, médiées et re-médiées.

Afin qu'une université devienne non seulement internationalisée, mais aussi multilingue, une politique linguistique claire est indispensable. La campagne interne viserait à convaincre des étudiants, des enseignants, des administrateurs que le plurilinguisme est non seulement utile, mais aussi une plus-value pour l'université qui peut leur permettre de comprendre que l'éducation internationale n'est donc pas seulement une question de standards et de mesures formels issus de la Déclaration de Bologne. L'éducation internationale concerne aussi la manière dont les différents acteurs feront pour que le local et l'international interagissent dans une nouvelle constellation de l'hybridité éducationnelle. 


\section{BIBLIOGRAPHIE}

Aigare, A., Thomas, P. L., \& Koyumdzhieva, T. (2011). Diversity Management in Higher Education Institutions: Key Motivators. Bachelor Thesis in Business Administration.

Airey, J. (2012) «I don't teach language». The linguistic attitudes of physics lectures in Sweden, AILA Review vol. 25, pp. 64-79.

Airey, J. (2011). «The Relationship between Teaching Language and Student Learning in Swedish University Physics. In In Bent Preisler, Ida Klitgard \& Anne H. Fabricius. (eds.) Language and Learning in the International University. From English Uniformity to Diversity and Hybridity. Bristol: Multilingual Matters, pp. 3-18.

Barbosa, I. \& Cabral-Cardoso, C. (2007). «Managing Diversity in Academic Organizations: A Challenge to Organizational Culture», Women In Management Review, vol. 22, n 4, pp. 274 -288.

Berthoud, A.-C. (2016). « Une communication scientifique plurilingue au service de la qualité des savoirs ». In Serge Borg et al. (sous la direction de) L'Université en contexte plurilingue dans la dynamique numérique. Paris: Edition s des archives contemporaines, pp. 167-174.

Berthoud, A.-C., Grin, F. \& Lüdi, G. (eds). (2013). Exploring the Dynamics of Multilingualism. Results from the DYLAN project. Amsterdam: John Benjamins.

Berthoud, A.-C. \& Gajo, L. (2016). « Le plurilinguisme comme révélateur de médiation et Instrument de re-médiation », dans Hommage à Liana Popp, Université de Cluj Napoca.

Bialystok E. (1999). «Cognitive complexity and attentional control in the bilingual mind». Child Development, vol. 70, n 3, pp. 636-644.

Bordons, M. \& Gómez, I. (2004). «Towards a single language in science? A Spanish view». Serials 17 (2) http://www.adawis.de/admin/upload/navigation/data/English\%20as\%20a\%20single\% 20language\%20of\%20Science\%20-\%20A\%20Spanish\%20view.pdf

Borg, S., Cheggour, M., Desrochers, N., Gajo, L., Larivière, V. et Vlad, M. (sous la direction de). (2016). L'Université en contexte plurilingue dans la dynamique numérique. Paris : Edition s des archives contemporaines.

Boyer, H. (1995). « De la competence ethnosocioculturelle », Le français dans le monde, vol. 272, pp. 41-44.

Bruchhagen, V. (2008) «Managing Gender \& Diversity : Eine kritische Gestaltungperspektive für den Profit- und Non-Profit-Bereich». In K. Iber \& B. Virtbauer (eds.), Diversity Management : Eine transdisziplinäre Herausforderung Göttingen : V\&R unipress, pp. 83-92.

Cancino, R. (2011). Language policy, language planning and language teaching at Danish universities. In R. Cancion, L. Dam \& K. Jaeger (eds.) Policies, Principles, Practices: New Directions in Foreign Language Education in the Era of Educational Globalization. New Castle upon Tyne, UK: Cambridge Scholars Publishers, pp. 138-160.

Castellotti, V. et Moore, D. (eds.) (1999.) Alternance des langues et construction des savoirs. Cahiers du français contemporain, Plurilinguisme et apprentissage. Lyon : ENS-Editions. 
Cattin, E. (2016). La représentation du plurilinguisme dans l'enseignement supérieur. Travail écrit de séminaire MA ‘Programme de spécialisation en analyse des discours et de la communication publics, Printemps 2016.

Cengiz, E. (2009). Diversity in Higher Education. Bachelor Thesis. Brugg: University of Applied Sciences Northwestern Switzerland.

Cenoz, J. (2009). Towards Multilingual Education: Basque Educational Research from an International Perspective. Bristol : Multilingual Matters.

Cenoz, J. (1997). « L'acquisition de la troisième langue : bilinguisme et plurilinguisme au pays basque ", Aile, vol. 10, pp. 159-175.

Coleman, J. A. (2006). English-Medium teaching in European higher education. Language Teaching, vol. 39, $\mathrm{n}^{\circ} 1$, pp. 1-14.

Cook V. (1997). «The consequences of bilingualism for cognitive processing». Tutorials in Bilingualism : Psycholinguistic Perspectives, pp. 279-300.

Chaplier, C. (2014). « Réflexions sur les enjeux et les conséquences de l'utilisation de l'anglais international », Educaçao, sociedade \& cultura, pp. 25-45.

Clarke, John, Thomas, P. \& Wallace, I. (s.d.) «How Should Diversity in the Higher Education System Be Encouraged?» Position Paper developed for the Business/Higher Education Round Table Summit Task Force. http://www.bhert.com/publications/discussion-papers/How-ShouldDiversity.pdf.

Derivry, M. (2011). « Enseignants de langue étrangère et internationalisation des marchés éducatifs et linguistiques : le cas de l'anglais et du français ». In Fred Dervin et Vasumathi Badrinathan (eds.), L'enseignement non natif: identités et légitimité dans l'enseignement-apprentissage des langues étrangères, Bruxelles : E.M.E, pp. 75-101.

Dervin, F. \& Badrinathan, V. (sous la dir. de) (2011). L'enseignement non natif: identités et légitimité dans l'enseignement-apprentissage des langues étrangères, Bruxelles : E.M.E.

Durkheim, E. ( $\left.{ }^{4} 1960\right)$. Les formes élémentaires de la vie religieuse, PUF.

Escudé, P. \& Gajo, L. (2016). Troisième partie. Axe 3. Langue unique ou modélisation du contact des langues dans l'université mondiale ?, In Serge Borg et al. (sous la direction de) L'Université en contexte plurilingue dans la dynamique numérique. Paris : Edition s des archives contemporaines, pp. 161-166.

Forster Vosicki, B. (2015). « Vers une approche institutionelle de la question du plurilinguisme à l'université ». In Xavier Gradoux, Jérôme Jacquin \& Gilles Merminod (eds.) Agir dans la diversité des langues. Mélanges en l'honneur d'Anne-Claude Berthoud, Louvain-la-Neuve, De Boeck, pp. 283-298.

Furlong, A. (2009). «The relation of plurlingualism/culturalism to creativity: a matter of perception», International Journal of Multilingualism, vol. 6, $\mathrm{n}^{\circ}$ 4, November 2009, pp. 343-368.

Gajo, L. (2013). « Le plurilinguisme dans et pour la science : enjeux d'une politique linguistique à l'université », Synergies Europe, vol. 8, pp. 97-110.

Gajo, L. (2005). «L'enseignant en section bilingue : un nouvel enseignant ?» Rencontres de l'enseignement bilingue francophone en Europe. Novembre 2005. Université de Prague. http:// rencontres-bilingues-prague.hautetfort.com/files/laurent_gajo.pdf.

Gajo, L., Grobet, A., Serra, C. Steffen, G., Müller, G. \& Berthoud, A.-C. (2013). «Plurilingualism and knowledge construction in higher education». In Anne-Claude Berthoud, François Grin \& Georges 
Lüdi (eds.) Exploring the Dynamics of Multilingualism. The DYLAN project. Amsterdam: John Benjamins, pp. 279-298.

García, O. \& Wei, L. (2014). Translanguaging: Language, Bilingualism and Education. New York: Palgrave Macmillan.

Graddol, D. (2006). «What will English look like in 2050?», Issues, vol. 149, 5-6.

Grosjean, François (2013). The Psycholinguistics of Bilingualism. Malden, MA: Wiley-Blackwell.

Hamel, E. (2013). L'anglais, langue unique pour les sciences ? Le rôle des modèles plurilingues dans la recherche, la communication scientifique et l'enseignement supérieur. Synergies Europe, vol. 8, pp. 53-66.

Hakuta K. \& Diaz R. M. (1985). « The relationship between degree of bilingualism and cognitive ability: a critical discussion and some new longitudinal data. », In Nelson K. E. Children's Language, vol. 5, pp. 320-344.

Holliday, A. (2006). «Native-speakerism», ELT Journal Vol 60/4. October 2006. Oxford University Press. Doi :10.1093/elt/cclo30.

Kekulé, A. (2010). «Soll Deutsch als Wissenschaftssprache überleben ? Contra : Der Zug ist abgefahren.» Zeit Online (http://www.zeit.de/wissen/2010-04/deutsch-forschungssprache/seite-2 )

Klaassen, R. (2008). «Preparing lectures of English-medium instruction». In R. Wilkinson \& V. Zegers (eds.), Researching Content and Language Integration in Higher Education, Nijmegen: Valkhof Pers, pp. 32-42.

Landemore, H. (2013). «Deliberation, cognitive diversity, and democratic inclusiveness: an epistemic argument for the random selection of representatives». Synthese, vol. 190, $\mathrm{n}^{\circ} 7$, pp. 1209-1231.

Llurda, E. (2005). Non-Native Language Teachers. Perceptions, Challenges and Contributions to the Profession. New York: Springer.

Lüdi, G. (2015). Monolingualism and multilingualism in the construction and dissemination of scientific knowledge. In Ulrike Jessner and Claire Kramsch (eds.) The Multilingual Challenge. Interdisciplanry perspectives. Berlin : de Gruyter Mouton, pp. 213-238.

Lüdi, G. \& Py, B. (2003). Etre bilingue. Berne : Peter Lang

Lüdi, G., Höchle, K. \& Yanaprasart, P. (2013). «Multilingualism and Diversity Management in Companies in the Upper Rhine Region». In Anne-Claude Berthoud, François Grin \& Georges Lüdi (eds.) Exploring the Dynamics of Multilingualism. The DYLAN project. Amsterdam: John Benjamins, pp. 59-82.

Lüdi, G., Höchle Meier, K. \& Yanaprasart, P. (eds.) 2016. Managing Plurilingual and Intercultural Practices in the Workplace. The Case of Multilingual Switzerland. Amsterdam: John Benjamins.

Marquardt, M. J. \& Horvath, L. (2001). Global Teams: How Top Multinationals Span Boundaries and Cultures with High-speed Teamwork. Mountain, CA: Davies-Black.

McMillan-Capehart, A. (2006). «Heterogeneity or Homogeneity: Socialization Makes the Difference in Firm Performance». Performance Improvement Quarterly, vol. 19, n 1, pp. 83-98.

Meek, V. L. \& Wood, F. Q. (1998). Managing Higher Education Diversity in a Climate of Public Sector Reform, Evaluations and Investigations Programme. Canberra: Evaluations and Investigations Program, Higher Education Division. 
Montgomery, C. «Developing Perceptions of Interculturality: A Troublesome Space?». In Bent Preisler, Ida Klitgard \& Anne H. Fabricius. (eds.) Language and Learning in the International University. From English Uniformity to Diversity and Hybridity. Bristol: Multilingual Matters, pp. 59-75.

Moore, D. (2006). Plurilinguismes et Ecole. Collection LAL. Paris: Didier.

Moring, T., Godenhjelm, S., Haapamäki, S., Lindström, J., Ostman, J.-O., Saari, M. \& Sylvin, J. (2013). «Language policies in universities and their outcomes: The University of Helsinki in a Northern European context.» In Anne-Claude Berthoud, François Grin, and Georges Lüdi (eds.) Exploring the Dynamics of Multilingualism. The DYLAN project. Amsterdam: John Benjamins, pp. 299-322.

Müller-Blaser, G. \& Pantet, J. (2008). « Plurilinguisme et constructions de savoirs disciplinaires dans l'enseignement universitaire », TRANEL, vol. 48, pp. 105-124.

Nussbaum, L., Moore, E. \& Borràs, E. (2013). «Accomplishing multilingualism through plurilingual activities». In Anne-Claude Berthoud, François Grin, and Georges Lüdi (eds.) Exploring the Dynamics of Multilingualism. The DYLAN project. Amsterdam: John Benjamins, pp. 229-252.

Olivas, M. A. (1992). «Legal norms in law school admissions: An essay on parallel universes». Journal of Legal Education, vol. 42, n 1, pp. 103-117.

Oltean, ş., Pop, L. Marga, D. \& Mihăescu, M. (2013). «Policies and practices of multilingualism at Babeş-Bolyai University (Cluj, Romania)». In Anne-Claude Berthoud, François Grin, and Georges Lüdi (eds.) Exploring the Dynamics of Multilingualism. The DYLAN project. Amsterdam: John Benjamins, pp. 323-342. Amsterdam: John Benjamins.

Peal, E. \& Lambert M. (1962). «The relation of bilingualism to intelligence». Psychological Monographs, vol. 75, n 546, pp. 1-23.

Phillipson, R. (1992). Linguistic Imperialism, Oxford: Oxford University Press.

Pierce, A. (2003). What does it mean to live in-between? Paper presented at the $4^{\text {th }}$ Annual IALIC Conference. Lancaster University, December 16, 2003.

Preisler, B., Klitgard, I, and Fabricius, A. H. (eds.) 2011. Language and Learning in the International University. From English Uniformity to Diversity and Hybridity. Bristol: Multilingual Matters.

Roussi, M. \& Cherkaoui Messin, K. (2011). «L'insécurité linguistique entre pratiques enseignantes et compétences langagières : vers une redéfinition du rôle de l'enseignant de langues ». In Fred Dervin \& Vasumathi Badrinathan. L'enseignement non natif: identités et légitimité dans l'enseignement-apprentissage des langues étrangères, Bruxelles : E.M.E, pp. 237-264.

Savin-Baden, M. (2008). Learning Spaces: Creating Opportunities for Knowledge Creation in Academic Life. Maidenhead : Open University Press.

Sauberer, G. (2008). « Über den Umgang mit sprachlicher Diversität. Diversity Management am Zentrum für Translationswissenschaft ». In Karoline Iber and Birgit Virtbauer (eds.) Diversity Management : Eine transdisziplinäre Herausforderung, Göttingen : V\&R unipress, pp. 29-38.

Schaller-Schwaner, I. (2009). «Under the Microscope: English for Plurilingual Academic Purposes». In Daniela Veronesi and Christoph Nickenig (eds.), Bi- and Multilingual Universities: European Perspectives and Beyond. Conference Proceedings, Bolzano-Bozen, 20-22 September 2007. Bozen-Bolzano University Press, pp. 245-263. 
Smit, U. (2003). English as lingua franca (ELF) as medium of learning in a hotel management educational program: an applied linguistic approach. VIEW - Vienna English Working Papers, vol. $12, \mathrm{n}^{\circ} 2$, pp. 40-74.

Steffen, G. (2013). Les disciplines dans l'enseignement bilingue. Apprentissage intégré des savoirs disciplinaires et linguistiques. Frankfurt am Main : Peter Lang.

Steffen, G. \& Pantet, J. (2011). « L'enseignement plurilingue au tertiaire : Regards croisés sur les représentations et les pratiques d'enseignement », Cahiers de l'ISIL, vol. 30, pp. 95-144.

Unterberger, B. (2012). «English-medium programmes at Austrian business faculties. A status quo survey on national trends and a case study on programme design and delivery», AILA Review, vol. 25, pp. 80-100.

Usunier, J.-C. (2010) « Un plurilinguisme pragmatique face au mythe de l'anglais lingua franca de l'enseignement supérieur ». In Les enjeux du plurilinguisme pour la construction et la circulation des savoirs, pp. 37-48. Bern : Schweizerische Akademie der Geistes- und Sozialwissenschaften.

Usunier, J.-C. (2013). «Plurilinguisme et construction des savoirs sous l'angle de diverses disciplines », Synergies Europe, vol 8, pp. 31-34.

Varghese, M., Morgan, B., Johnson, B. \& Johnson, K. (2005). «Theorizing language teacher identity: three perspectives and beyond», Journal of Language, Identity and Education, vol. $4, \mathrm{n}^{\circ} 1$, pp. 1-44.

Veronesi, D., Spreafico, L. Varcasia, C., Vietti, A. \& Franceschini, R. (2013). «Multilingual higher education between policies and practices: A case study». In Anne-Claude Berthoud, François Grin, and Georges Lüdi (eds.) Exploring the Dynamics of Multilingualism. The DYLAN project. Amsterdam : John Benjamins, pp. 253-278.

Vygotski, L. (1997). Pensée et langage. Paris : La Dispute.

Wächter, B. \& Maiworm, F. (2008). English-Taught Programmes in European Higher Education. The Picture in 2007. Bonn: Lemmens.

Wilkinson, R. (2010). English-medium instruction at a Dutch university: What have we learned from a quarter of a century experience? Paper presented at The Norwegian Forum for English for Academic Purposes (NFEAP), Oslo.

Yanaprasart, P. (2012). «Plurilinguisme institutionnel comme mesure de gestion de la diversité linguistique. Quel atout interculturel du plurilinguisme individuel ? ». In Eliane Costa-Fermandez \& Odette Lescarret (eds). De la diversité linguistique aux pratiques interculturelles. Paris :

L'Harmattan, pp. 229-241.

Yanaprasart, P.(2015a) «Diversity management practices: measures and indicators». Scientific Report. Swiss National Scientific Foundation, September 2015.

Yanaprasart, P. (2015b) «Multilinguaculturaling: making an asset of multilingual human resources in organizations». In Nigel Holden, Snejina Michailova \& Susanne Tietze, The Routledge Companion to Cross-Cultural Management. London: Routledge Companions, pp. 112-130

Yanaprasart, P. (2016a). «The Challenge of the Management of Diversity». In Lüdi, Georges, Katharina Höchle Meier \& Patchareerat Yanaprasart (eds.) Managing Plurilingual and Intercultural Practices in the Workplace. The Case of Multilingual Switzerland. Amsterdam: John Benjamins.

Yanaprasart, P. (2016b) «Managing Language Diversity in the Workplace: Between 'One Language Fits All' and 'Multilingual Model in Action'. Universal Journal of Management. (ISSN: 2331-9577) 
Yanaprasart, P. \& Lüdi, G. (2017) «Diversity and multilingual challenges in academic settings». International Journal of Bilingual Education and Bilingualism, http:// dx.doi.org/10.1080/13670050.2017.1308311, Routledge Taylor \& Francis Group.

Yanaprasart, P. \& Mélo-Pfeifer, S. (2017) Représentations des enseignants plurilingues par des étudiants dans le domaine de la didactique des langues. Entre perception monolingue et plurilingue du plurilinguisme, Projet concours interne Genève - Hambourg, février-juillet 20117.

Zhu, H. (2014) «Piecing together the 'workplace multilingualism' jigsaw puzzle». Multilingua, vol. 33, n 1-2, pp. 233-242.

\section{NOTES}

1. Benefits and Challenges of Diversity in Academic Settings http://wiseli.engr.wisc.edu/docs/ Benefits_Challenges.pdf, consulté mars 2017.

2. Processus qu'Usunier (2010) relie à un mouvement de marchandisation de l'enseignement supérieur.

3. https://www.swissuniversities.ch/fr/organisation/projets-et-programmes/ (consulté avril 2017)

4.

https://www.unil.ch/central/files/live/sites/central/files/docs/

plan_intentions_unil_12_16.pdf (consulté avril 2017)

5. Selon le Cadre européen commun de référence pour les langues.

6. http://www.observatoireplurilinguisme.eu/fr/ (consulté avril 2017).

\section{RÉSUMÉS}

Ces dernières années, les processus d'internationalisation de la formation et de la recherche ont accéléré le développement et la promotion de l'anglais comme langue internationale pour les sciences et pour l'enseignement/apprentissage. La question développée dans cet article concerne moins l'impérialisme linguistique de l'anglais dans le milieu académique globalisé que la perception de l'usage de la langue par ceux qui l'utilisent. Qui l'emploie avec qui, pour quelle raison, dans quel contexte, à quel moment et de quelle manière? Comment chaque choix est encadré, soutenu, géré, (dé)valorisé, outillé, médié ou remédié ? Quelle politique linguistique universitaire à mettre en place pour trouver un juste équilibre entre l'anglais « lingua academica » et les autres langues, dans le but de garantir une convergence entre l'internationalisation de la formation et le maintien de la diversité au niveau individuel et institutionnel, ce en termes de complémentarité et d'intégration? L'objectif de cette contribution sera de confronter les représentations sociales que les acteurs sociaux ont de l'internationalisation avec la politique linguistique institutionnelle plurilingue, d'explorer ce que représentent les pratiques scientifiques et académiques plurilingues et d'identifier une convergence ou divergence possibles entre les connaissances scientifiques et la sensibilité des acteurs aux théories sur le plurilinguisme. Notre intérêt porte particulièrement sur les enjeux linguistiques dans le processus d'internationalisation des savoirs, notamment sur le rôle de (re-)médiation du langage et les fonctions des langues tels rapportés par des étudiants de différentes disciplines. 
Currently, the process of internationalization of training and research has accelerated the development and the promotion of English as an international language for science and teaching/learning. The question developed in this article is less concerned with the linguistic imperialism of English in the globalized academic environment than it is with the perception of the language use by those who employ it. Who uses it with whom, for what reason, in what context, at which particular moment time and in what way? How is each choice framed, supported, managed, (de)valorized, instrumented, mediated or remedied? How does a university put in place its language policy so as to find a fair balance between English «Lingua Academica» and other languages, with the aim of ensuring a convergence between the internationalization of the education and the maintenance of diversity at both the individual and institutional levels, in terms of complementarity and integration? The goal of this contribution is to confront the social representations that the social actors have regarding internationalization in multilingual institutional linguistic policies, to explore the meaning of plurilingual scientific and academic practices, and also to identify a possible convergence or divergence between scientific knowledge and the sensitivity of the actors towards theories on plurilingualism. Our interest focuses particularly on language issues in the process of knowledge internationalization, including the role of (re-)mediation of the language and the functions of languages such as reported by students of different disciplines.

\section{INDEX}

Mots-clés : enseignement supérieur, plurilinguisme, internationalisation, diversité académique, (re-)médiation

Keywords : higher education, multilingualism, internationalization, academic diversity, (re)mediation 